\title{
Experimental Study of a Colloidal Damper to Practical Application*
}

\author{
Takuzo IWATSUBO ${ }^{* *}$, Kazuhiro WASHIO ${ }^{* * *}$, Hiroki YANO ${ }^{* * * * *}$ \\ and Mitsuru MIYAZAKI ${ }^{* * * * *}$ \\ **Department of Mechanical System Engineering, Kansai University, \\ 3-3-35 Yamate-cho, Suita-shi, Osaka, 564-8680, Japan \\ E-mail: iwatsubo@ipcku.kansai-u.ac.jp \\ ${ }^{* * *}$ Graduate School of Engineering, Kansai University, \\ 3-3-35 Yamate-cho, Suita-shi, Osaka, 564-8680, Japan \\ E-mail: sa6m158@ipcku.kansai-u.ac.jp \\ ${ }^{* * * *}$ Former Department of Mechanical System Engineering, Kansai University, \\ 3-3-35 Yamate-cho, Suita-shi, Osaka, 564-8680, Japan \\ *****Research \& Development Division, Oiles Corporation, \\ 1000 Hakari-cho, Asahiga-shi, Tochigi, Japan \\ E-mail:m.miyazaki@oiles.co.jp
}

\begin{abstract}
A colloidal damper is a damping element that has a novel structure which is fusing technology of mechanical engineering and nanotechnology. This structure is composed of cylinder and piston in which a mixture of hydrophobic porous matrix and liquid are inserted. If an external force impresses to the damper, the pressure in the cylinder increases, and then the liquid flows into the pores of porous matrix. In this time the surface extension force for compression is larger than that for relaxation. This difference of the surface extension force produces a damping energy.

The colloidal damper which was applied the difference of surface extension of compression and relaxation was first developed by Eroshenko. In his device the damper had colloidal liquid and oil in the bag in the cylinder, and damping characteristics and kinds of the colloid had not studied. A simple damper composed of piston and cylinder was made to investigate the basic static characteristics of the colloidal damper in our group. Then influence of hydrophobic coatings on dynamic characteristics and endurance of coatings of the silica gel were investigated. When the damper is used in practice, there are many applications. So, effect of size of the damper should be investigated. But effect of size on the damping has not been investigated. In addition, investigation for the single cylinder type damper has been mainly studied, but this type need bias pressure to have a damping force in the negative force range. In order to avoid this disadvantage, double cylinder type damper was developed. Then in this paper the relationship between the stroke and the pressure, the dissipative energy and the efficiency are investigated for the single colloidal damper and the double colloidal damper, and dynamic characteristics of these dampers were compared. Additionally, mass-spring-damper system in which the single colloidal damper is installed is designed for the suspension of a quarter of a car to demonstrate a practical use.
\end{abstract}

Key words: Damper, Colloidal Damper, Hydrophobic Silica Gel, Car, Suspension 


\section{Introduction}

The viscous damper is well known as a machine element to dissipate the energies of shock and vibration by using fluids flowing in narrow holes ${ }^{(1),(2)}$. Hydraulic damper (HD) dissipates the mechanical energy by disturbing the hydraulic oil to pass through small holes. During a Poiseuille flow, the viscous force produces dissipation of the mechanical energy. The dissipate energy depend on the viscosity of the oil and the piston velocity. This mechanism is called viscous dissipation.

On the other hand, colloidal damper (CD), proposed by Eroshenko ${ }^{(3),(4)}$, have a cylinder piston construction, but no holes are machined in the piston; a mixture of the hydrophobic porous matrix, e.g., modified silica gel, and its associated liquid, e.g., water, produce the colloid, which replaces the hydraulic oil. The mechanism of the energy dissipation of CD is when the liquid flow into the pores by acting external force, the work by the surface extension in the small holes of the silica gel dissipates the energy ${ }^{(5)}$. Both HD and CD dissipate the mechanical energy by forcing the working fluid to pass through narrow orifices. The difference between them is the size of the orifices, which is principle of viscosity for $\mathrm{HD}$ and surface extension for $\mathrm{CD}$. In addition, $\mathrm{CD}$ dissipation is not depending on the piston velocity, because of surface extension and its efficiency is higher than HD.

In the previous work, influence of pre pressure, and hydrophobic coating of silica gel and so on were investigated for static and dynamic tests of the single $\mathrm{CD}$, respectively; thereby the dissipative energy is increased as decrease of pre pressure, and the best hydrophobic material were known ${ }^{(6),(7),(8)}$. However, specific characteristics are not compared by the difference of the $\mathrm{CD}$ mechanism.

In this paper, the relationship between the stroke and the pressure, the dissipative energy and the efficiency are investigated for the single and the double CDs, and dynamic characteristics of these dampers were compared. Additionally, mass-spring-damper system in which the single $\mathrm{CD}$ is installed is designed for the suspension of a quarter of a car to demonstrate a practical use of $\mathrm{CD}$. And the vibration characteristic was examined for the single degree of freedom model, and the possibility of the practical use of $\mathrm{CD}$ is investigated.

\section{Dynamic test rig of colloidal damper}

Single CD which has one cylinder and double $\mathrm{CD}$ which has two cylinders are shown in Fig.1 (a) and (b) respectively. As CD is effective for only pressuring stroke, it is difficult to have a dissipative force for fore and back strokes. In order to complicate this disadvantage, $\mathrm{CD}$ with two cylinders is designed, which is called double colloidal damper. $\mathrm{CD}$ has the problem of causing negative pressure in a negative stroke area. This problem is solved by giving pre pressure to the cylinder with the pump.

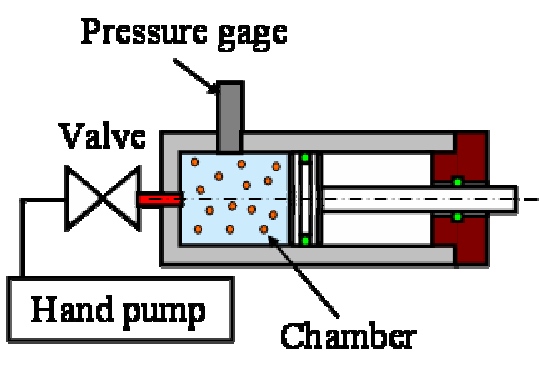

(a) Single colloidal damper

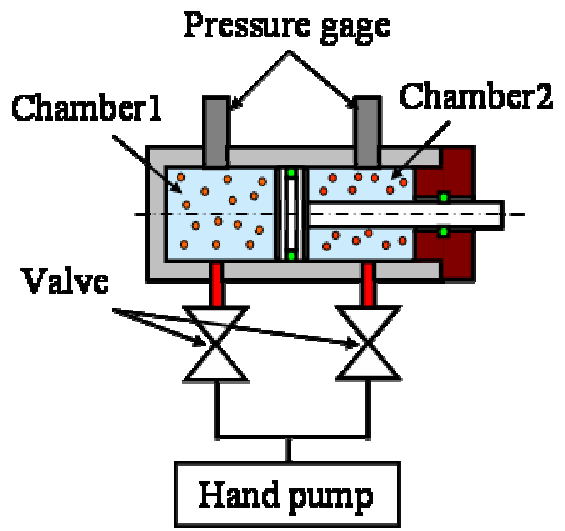

(b) Double colloidal damper

Fig. 1 Colloidal damper 
In the single $\mathrm{CD}$, diameter of piston is $8 \mathrm{~mm}$; thread plugs and high pressure gauge close the test chamber. Copper gaskets, $\mathrm{O}$ ring and $\mathrm{V}$ packing are used to seal the experimental chamber, in which can be created a maximum pressure of 60Mpa. In double CD, diameter of piston head is $20 \mathrm{~mm}$, diameter of piston rod is $14 \mathrm{~mm}$, and piston head area of chamber 1 side is $314 \mathrm{~mm}^{2}$ and that of chamber2 is $160 \mathrm{~mm}^{2}$. Thread plugs and high pressure gauge close the each chamber. Copper gaskets, $\mathrm{O}$ ring and $\mathrm{V}$ packing are used to seal the experimental chamber, in which can be created a maximum pressure of $45 \mathrm{Mpa}$. The amounts of modified silica gel and water are $1 \mathrm{~g}$ and $2 \mathrm{~g}$, respectively.

Fig. 2 shows the dynamic test rig of $C D$ to obtain dynamic characteristics of single and double colloidal dampers, where relation between stroke and pressure in the chamber are measured. A high-power vibration is produced by the electric vibration exciter, which acts directly to the piston. Maximum force produced by the exciter is $F_{\max }=14700 \mathrm{~N}$; frequency range provided by vibrator is $2-3000 \mathrm{~Hz}$ and the maximum stroke is $56 \mathrm{~mm}$ (peak - peak) for the single CD and 40mm ( $\mathrm{p}-\mathrm{p}$ ) for the double CD. In each chamber, the amounts of modified silica gel and water are $1 \mathrm{~g}$ and $19 \mathrm{~g}$, respectively.

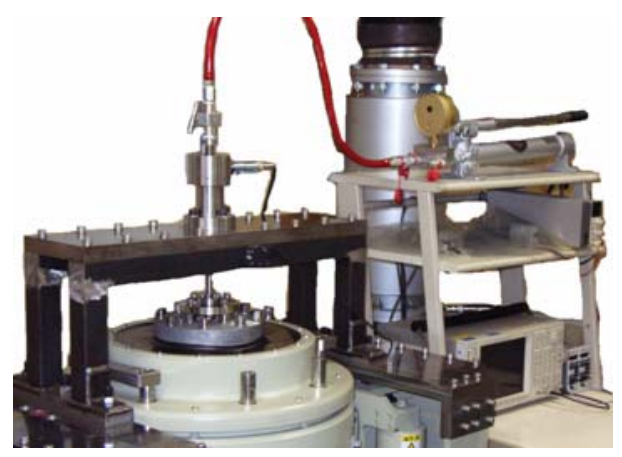

(a) Over view of experimental apparatus

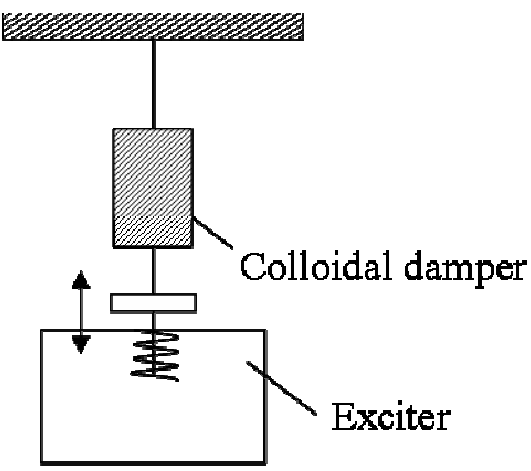

(b) Test rig model

Fig. 2 Dynamic test rig of colloidal damper

Fig.3 shows a measuring system and data acquisition system. The displacement of piston is measured by laser displacement sensor and the pressure of the piston chamber is measured by the high pressure gauge. These data are sent to PC to display the pressure versus stroke of the piston and are printed.

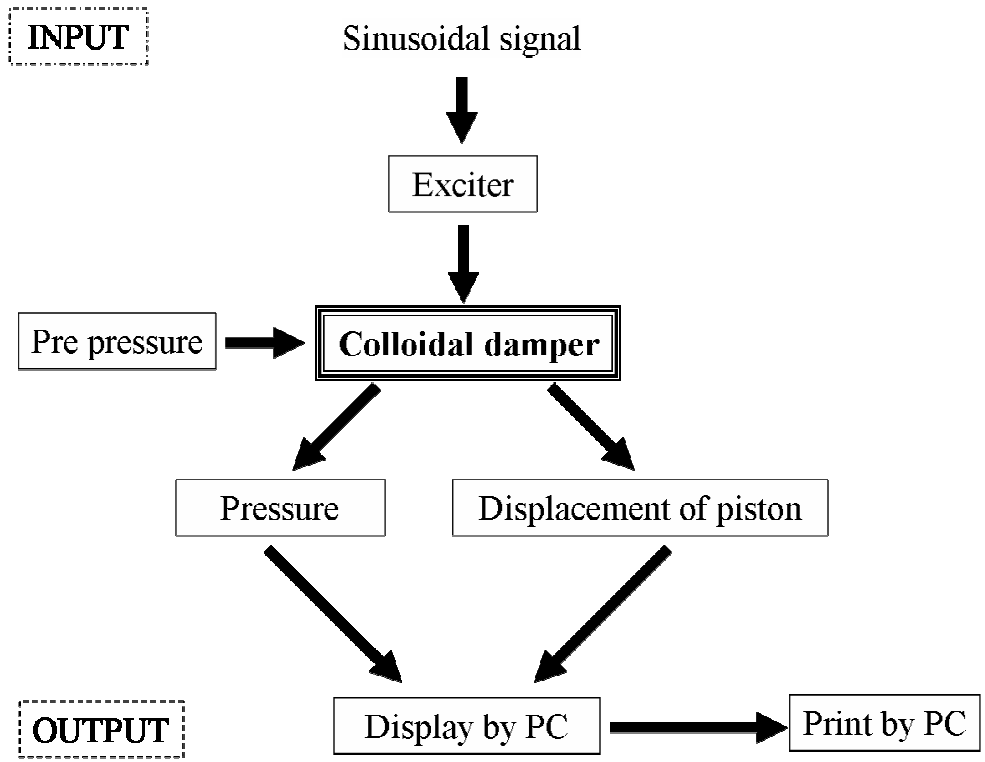

Fig. 3 Measuring systems 


\section{Dynamic characteristics of colloidal damper}

Fig.4 shows a typical hysteresis curve of CD. (1) is dead zone, where the piston strokes in the cylinder but the pressure in the cylinder does not increase. This phenomenon is occurred both in static and dynamic tests, but the reason is not known yet. Fig.4 (2) (4) become lines that look like the pressure change when water is compressed in the parts where the water exists in the pores of the silica gel. And there parts are called the 2nd to 4th branches. The energy dissipation with silica gel is done in these parts. The water configurations in the pore are shown in Fig.4 (a) (2) (4), where the 2nd branch is beginning the water into the pore, so energy dissipation is hardly done by changing the contact angle from 0 to 90 degree. The 3 rd branch is the part where the water is entering into the pore, so energy dissipates is constant. The 4th branch shows that the water is felled in the pore and compressed the air in the pore. (5) in Fig.4 is the pressure change that begins decompressing is called the 5 th branch. The inclination of this 5 th branch is represented by difference of the contact angle. The hysteresis curve is changed by the stroke of the piston as shown in Fig.4 (b) (d).
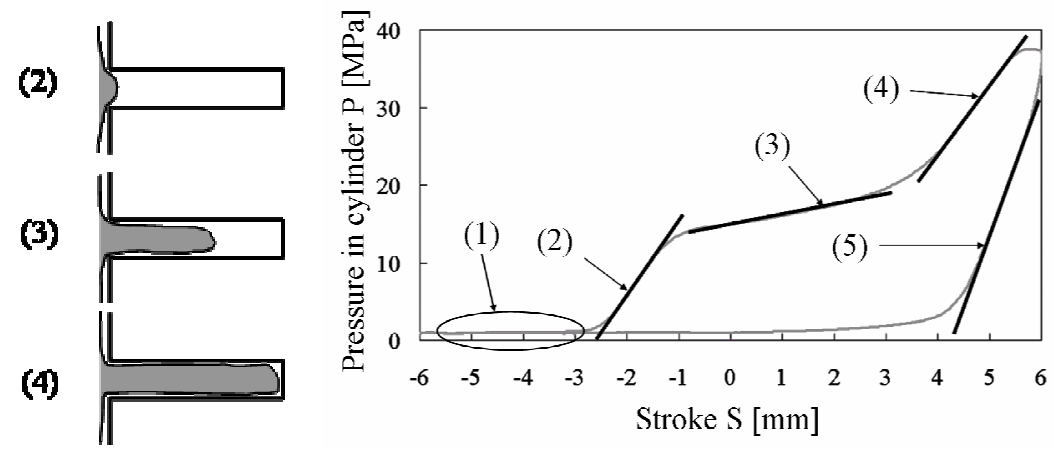

(a) Hysteresis curve and state of the water in the pore

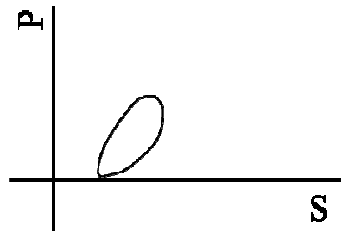

(b) 2nd branch

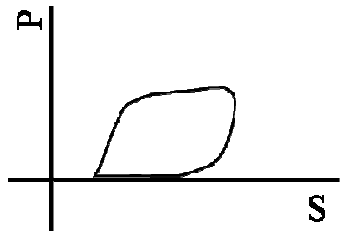

(c) 3rd branch

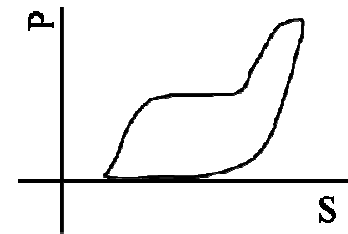

(d) 4th branch

Fig. 4 Hysteresis curve

\subsection{Characteristics of single CD}

Fig. 5 shows the hysteresis curve of the single $C D$, in which displacement of piston and loading frequency are fixed. Upper and lower curves of the hysteresis show compression and relaxation cycles, respectively, so the cycle rotates clock wise direction. Pre pressure is supplied 4MPa and 12MPa for Figs.5 (a) (b) and (c) (d) respectively, so beginning of histeresis curve is changed. Figs.5 (a) and (b) show hysteresis curve of the 1st and 2nd branches in Fig.4 and Figs.5 (c) and (d) shows that of the 1st to 3rd branch.

When Figs.5 (a) and (c) are compared with Figs.5 (b) and (d), the difference according to the frequency is known, that is, the inclination of the 5th branch of the hysteresis becomes large and areas of the hysteresis become large as increase of the load frequency. That is considered that the air contained in the water is affected. When Fig.5 (a) and (b) are compared with Fig.5 (c) and (d), the effect of pre pressure in cylinder is known that a lot of energy is dissipated as increase of the pre pressure. This is because the 1st branch (dead zone area) is decreased and the 3rd branch of the hysteresis is utilized. It is know from this result that pre pressure is important to obtain a large hysteresis curve and the area of hysteresis become large as increase of frequency. 


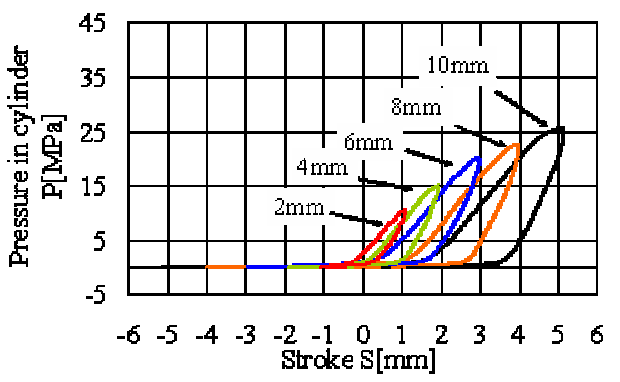

(a) Pre pressure: $4 \mathrm{MPa}$, Frequency: $5 \mathrm{~Hz}$

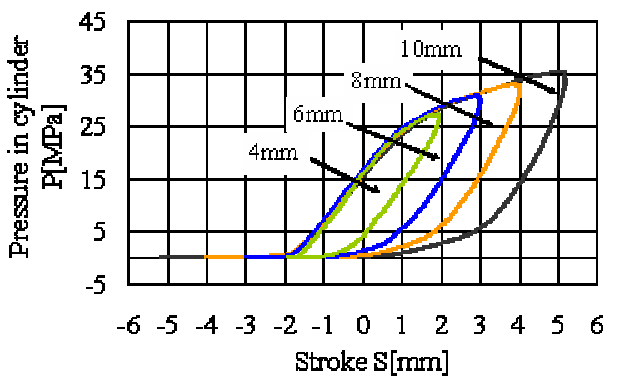

(c) Pre pressure: $12 \mathrm{MPa}$, Frequency: $5 \mathrm{~Hz}$

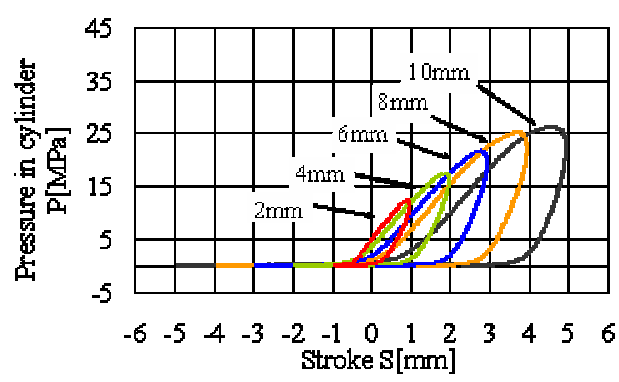

(b) Pre pressure: 4MPa, Frequency: $9 \mathrm{~Hz}$

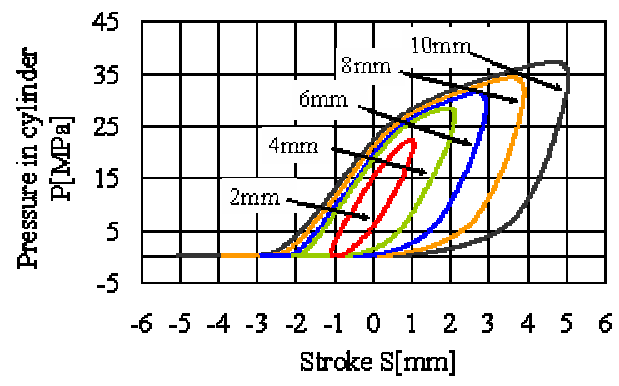

(d) Pre pressure: 12MPa, Frequency: $9 \mathrm{~Hz}$

Fig. 5 Hysteresis curve of single colloidal damper

Fig.6 (a) shows the relation between the cylinder pressure (working pressure) and dissipative energy of the single $\mathrm{CD}$. As the pressure is increased, the dissipative energy increased. In the case of $\mathrm{HD}$, the dissipative energy depends on piston velocity, but it depends on the pre pressure of the cylinder in the CD.Fig.6 (b) shows the relation between the maximum cylinder pressure and efficiency of the single $\mathrm{CD}$, where the efficiency is defined as the ratio of the area of hysteresis cycle to the area of under part of the upper hysteresis curve. The efficiency is increased as increase of the cylinder pressure and the loading frequency.

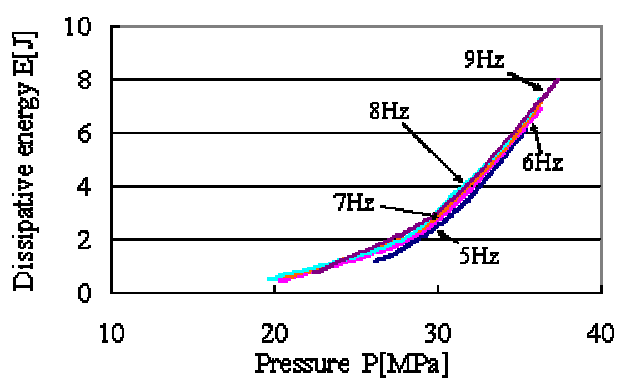

(a) Dissipative energy

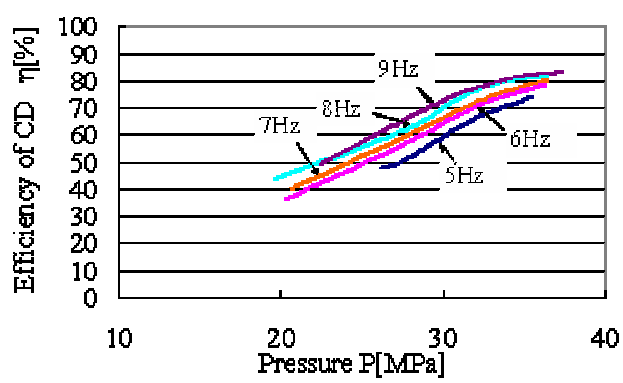

(b) Efficiency of the single CD

Fig.6 Dissipative energy and efficiency of single CD (pre pressure: 12MPa)

\subsection{Characteristics of double CD}

Fig. 7 shows the hysteresis curve of the double CD. When the pressure of the chamber1 becomes positive, the force of the piston is defined to be positive. The shape of the hysteresis of chamber1 and chamber2 is different from the same stroke. The reason is that both cross sections of the chambers of the cylinder are different; therefore volume change ratios in both cylinders are different. The volume change ratio of the cylinder of the small section area is larger than that of the large section area. Therefore pressure of chamber2 become larger than that of chamber1 


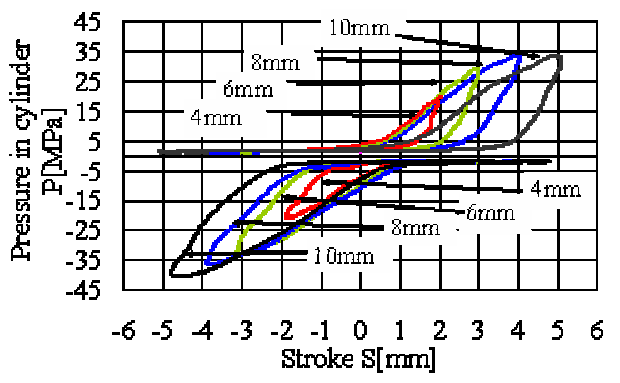

(a) Pre pressure: $2 \mathrm{MPa}$, Frequency: $5 \mathrm{~Hz}$

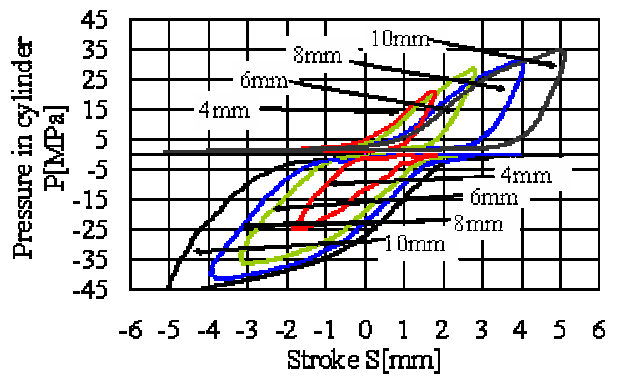

(c) Pre pressure: $8 \mathrm{MPa}$, Frequency: $5 \mathrm{~Hz}$

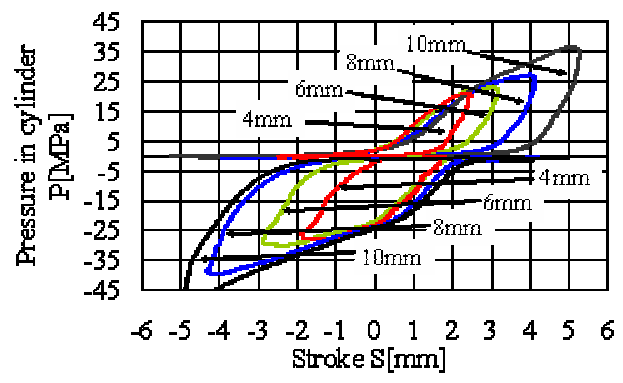

(b) Pre pressure: $2 \mathrm{MPa}$, Frequency: $7 \mathrm{~Hz}$

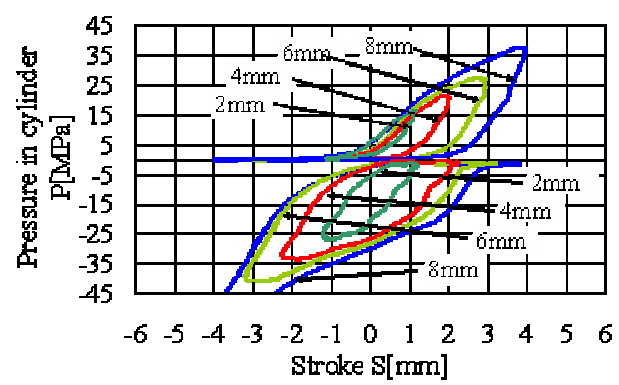

(d) Pre pressure: $8 \mathrm{MPa}$, Frequency: $7 \mathrm{~Hz}$

Fig.7 Hysteresis curve of double colloidal damper

Fig. 8 shows the relation between stroke and dissipative energy of the double $\mathrm{CD}$. When chamber1 is compared with chamber2, chamber2 dissipates a lot of energy. The dissipative energy is inverse proportion to the area of the piston head.

Fig.9 shows the relation between stroke and efficiency of the double CD. When chamber1 is compared with chamber2, efficiency of chamber2 is larger than that of chamber1. The phase difference between input force and output pressure is increased, as increase of the frequency in both chambers, and the efficiency of the damping is increased.

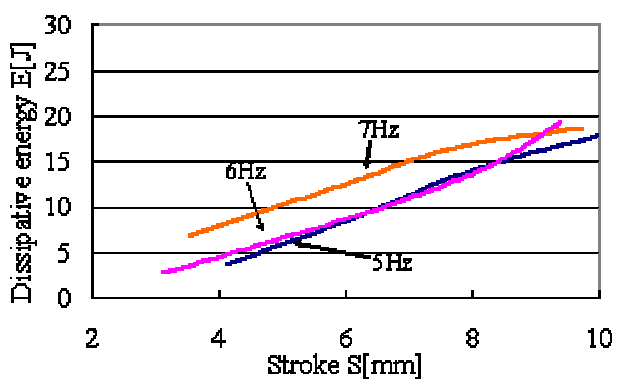

(a) Pre pressure: $2 \mathrm{MPa}$, chamber 1

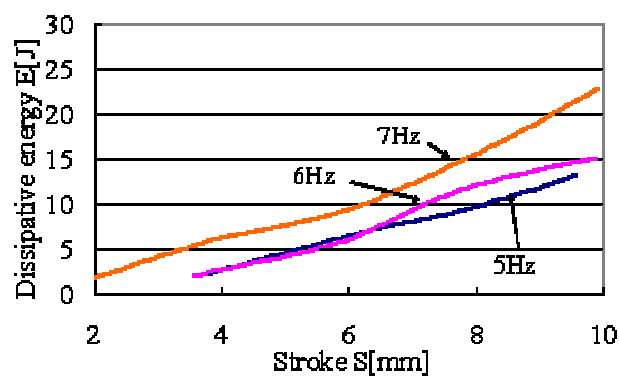

(b) Pre pressure: $2 \mathrm{MPa}$, chamber2

Fig. 8 Relation between stroke and dissipative energy of the double CD

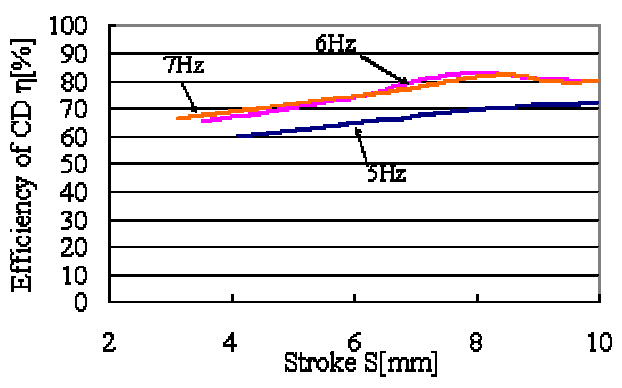

(a) Pre pressure: $2 \mathrm{MPa}$, chamber 1

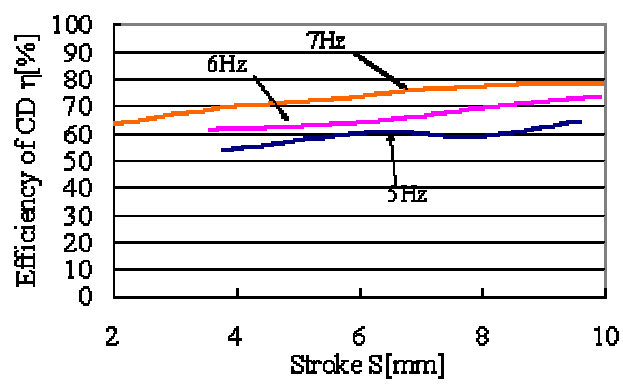

(b) Pre pressure: $2 \mathrm{MPa}$, chamber2

Fig.9 Relation between stroke and efficiency of the double CD 


\subsection{Comparison of performance of the two CDs}

In the $\mathrm{CD}$ the dissipative energy is proportion to the section area of the piston head. The dissipative energy per unit cross-sectional area are shown in Fig.10, where the relation between cylinder pressure of each chamber and dissipative energy are shown unit cross-sectional area. In the double $\mathrm{CD}$, dissipative energy of chamber1 and chamber2 approaches by increasing of pre-pressure, and both chamber's dissipative energy for each unit sectional area of pre-pressure $8 \mathrm{MPa}$ becomes almost equal. The dissipative energy for each unit cross-sectional area of single $\mathrm{CD}$ is the largest of the three.

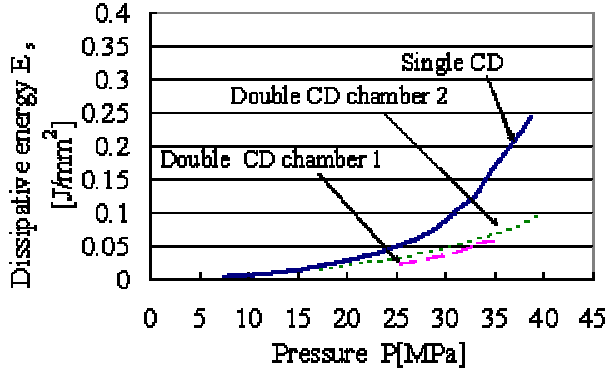

(a) Pre pressure: $2 \mathrm{MPa}$, Frequency: $6 \mathrm{~Hz}$

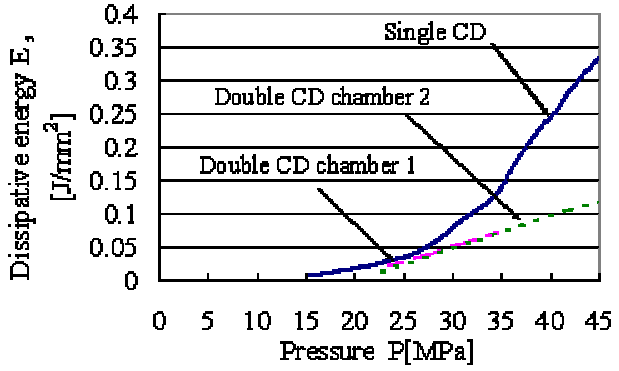

(b) Pre pressure: $8 \mathrm{MPa}$, Frequency: $6 \mathrm{~Hz}$

Fig.10 Relation between cylinder pressure and dissipative energy per unit cross-sectional area

\section{Application of CD for car suspension}

In modeling the car, one degree of freedom model which composed of mass, spring, and damper is used. Because of investigation of damping characteristics of the single CD suspension, the spring and the damping of the tire, mode of rolling and pitching oscillation are excluding. The mass-spring-damper system in which the single CD is installed for the suspension of a quarter of a car is shown in Fig.11. In this system pre pressure is supplied by the weight instead of pump, and the weight is $250 \mathrm{~kg}$, spring coefficient of spring 1 and spring2 are $9.8 \mathrm{kN} / \mathrm{m}$ and $19.6 \mathrm{kN} / \mathrm{m}$ respectively, diameter of piston is $11.2 \mathrm{~mm}$, the maximum pressure of the cylinder is $60 \mathrm{MPa}$. Maximum force produced by the exciter is $F_{\max }=5880 \mathrm{~N}$; frequency range provided by exciter is $2-500 \mathrm{~Hz}$ and the maximum stroke is $25 \mathrm{~mm}$. (peak - peak) The amounts of modified silica gel and water are $3 \mathrm{~g}$ and $7 \mathrm{~g}$, respectively.

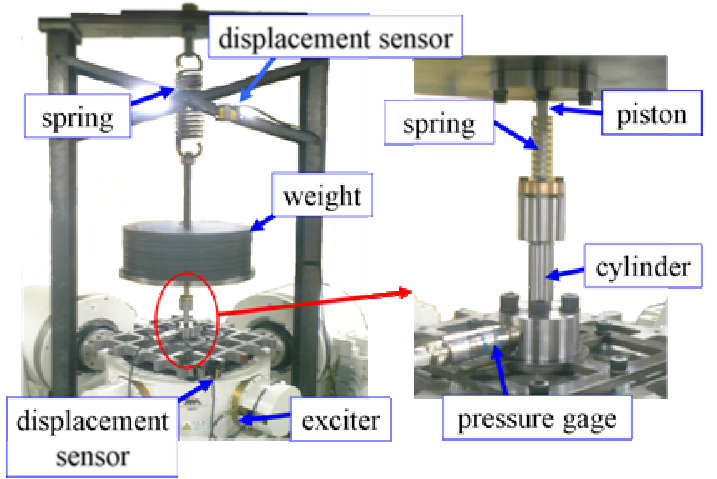

(a) Over view of new experimental apparatus

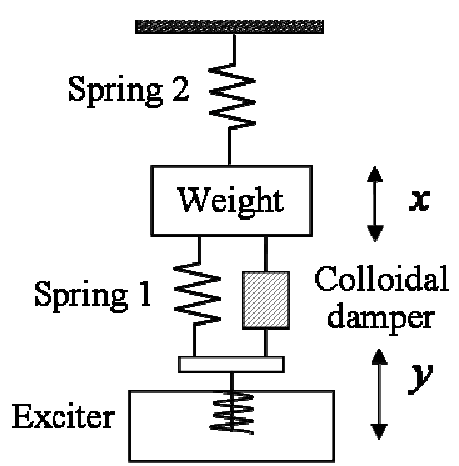

(b) model of test rig

Fig.11 Mass-spring-damper system of CD

Fig. 12 shows the time history of the exciting table, the weight, stroke and pressure for exciting frequency $=4,6,10,12 \mathrm{~Hz}$ and exciting amplitude $=4 \mathrm{~mm}$, and Fig. 13 shows the hysteresis curve of pressure versus stroke of the damper for the exciting amplitude $2 \mathrm{~mm}$, $4 \mathrm{~mm}, 6 \mathrm{~mm}$. 


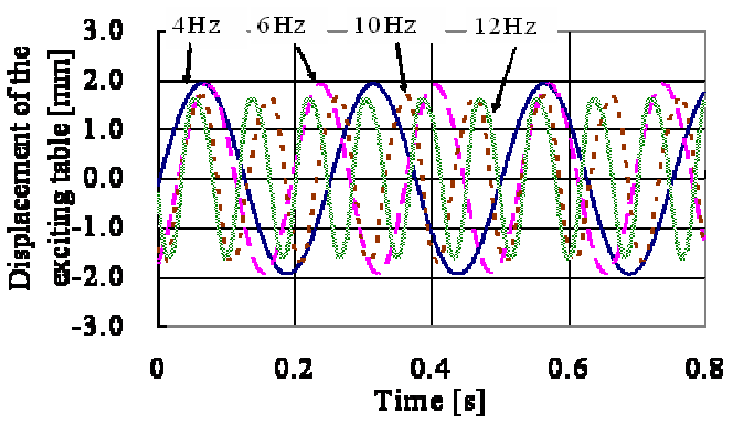

(a) Input amplitude

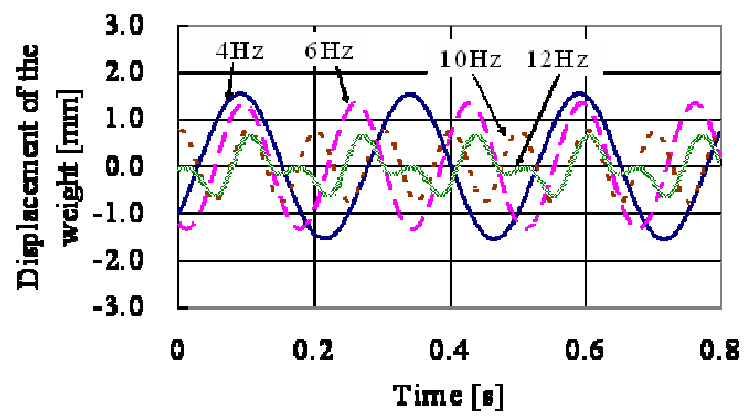

(b) Output amplitude

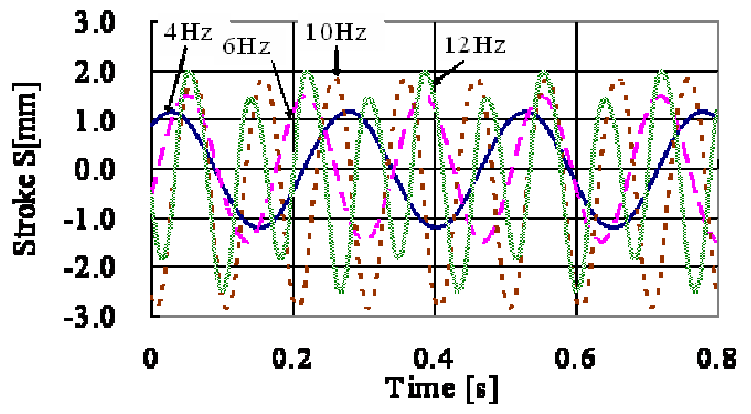

(c) Stroke

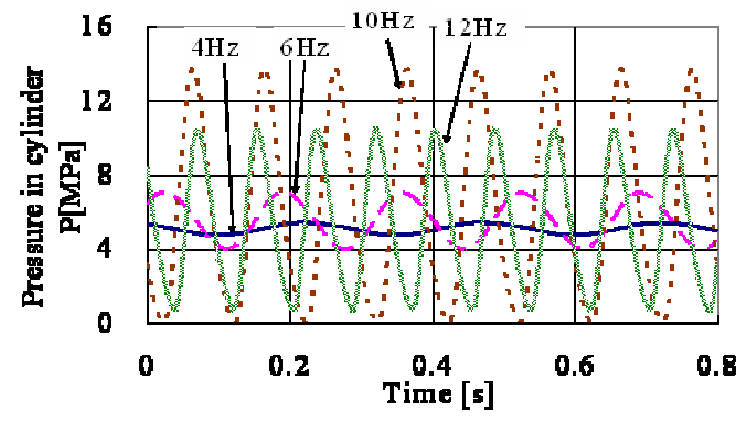

(d) Pressure

Fig.12 Time history

Fig. 13 shows hysteresis curve of single CD for car suspension. From these results, it is known that the pressure of the damper did not increase for low frequency region, since the friction of V-packing which was used in the seal of a connecting rod of the piston was very large and, thus, the connecting rod was fixed. The pressure began to increase at $10 \mathrm{~Hz}$ for the exciting amplitude $2 \mathrm{~mm}$, at $8 \mathrm{~Hz}$ for $4 \mathrm{~mm}$ and at $6 \mathrm{~Hz}$ for $6 \mathrm{~mm}$. These are as the inertia force was increased as increase of the exciting frequency; the inertia force becomes larger than the friction force of the V-packing. The amplitude becomes large at $10 \sim 12 \mathrm{~Hz}$, so this frequency range may be resonance region. 


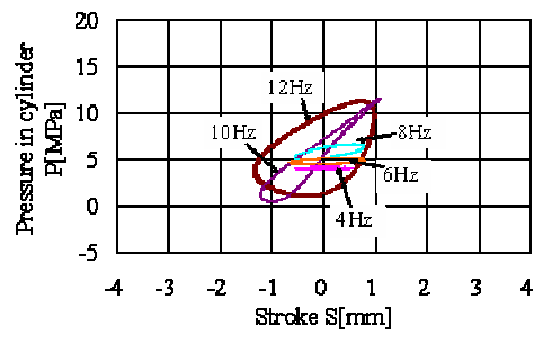

(a) Exciting amplitude: $2 \mathrm{~mm}$

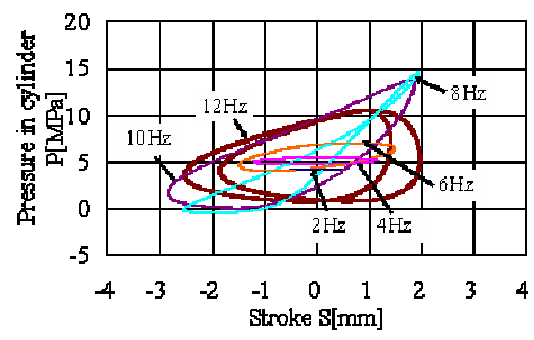

(b) Exciting amplitude: $4 \mathrm{~mm}$

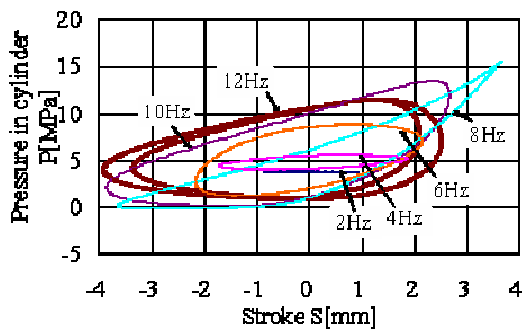

(c) Exciting amplitude: $6 \mathrm{~mm}$

Fig.13 Hysteresis curve of single CD for car suspension

Fig.14 shows the amplitude ratio of the weight (x) to the exciting table(y), Lissajous at the maximum amplitude, phase lag and amplitude ratio of the stroke (S) to the exciting table (y).

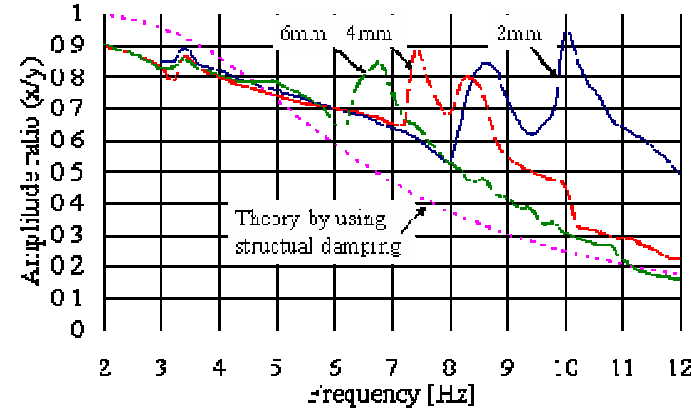

(a) Amplitude ratio of $x$ to $y$

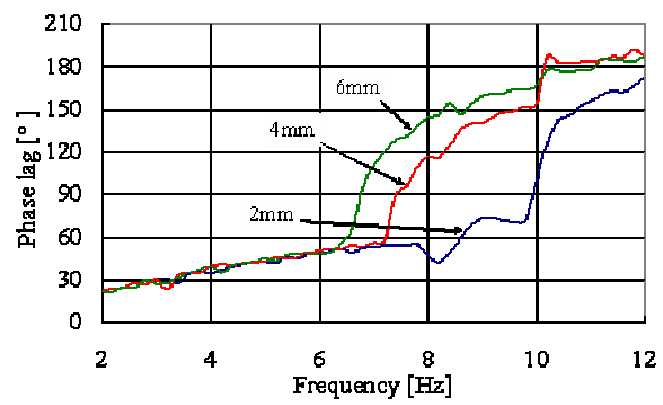

(c) Phase lag

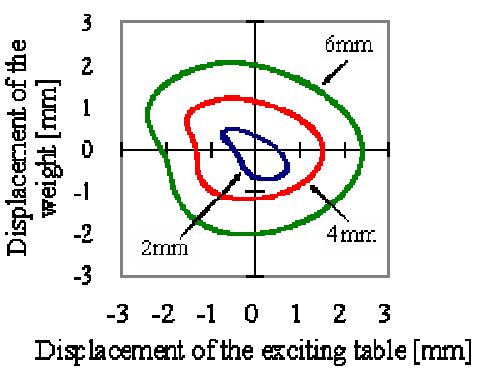

(b) Lissajous

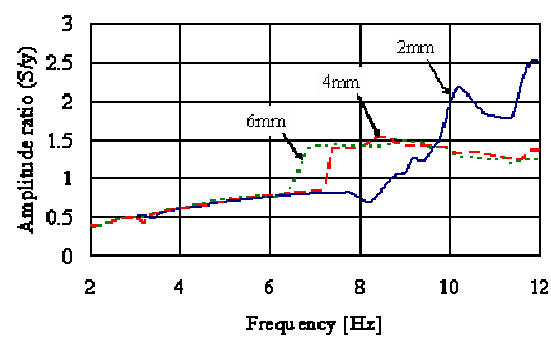

(d) Amplitude ratio of $\mathrm{S}$ to $\mathrm{y}$

Fig.14 Amplitude ratio, Lissajous and Phase lag

Fig.14 (a) shows that the amplitude ratio decreases as increase of the exciting frequency. Damping energy induced by the $\mathrm{CD}$ increases as increase of the exciting frequency. Fig.14 (b) shows that Lissajous is almost circle and Fig.14 (c) shows the phase lag between the weight and the exciting table under the constant exciting displacement. From these results the amplitude of the weight decreases and phase delay angle approaches 180 degree as increase of frequency. CD's stroke is decided by relation of absolute amplitude and phase lag. CD's stroke (dissipative energy) increases as increase of frequency 
until phase delay angle approaches 180 degree. On the other hand, CD's stroke slightly decreases as decrease of the amplitude of the weight when phase delay angle is vicinity of 180 degree. Therefore the CD is effective as a damper of the mass-spring-damper system.

\section{Conclusion}

This paper studied on the dynamic characteristics of the single CD and the double CD and on the characteristics of a single $\mathrm{CD}$ which is installed in a mass-spring-damper system in order to investigate the possibility for the suspension of a quarter of a car. The results are follows;

(1) The dissipative energy of CD increases as the working pressure (stroke) increases, and increases slightly as increase of frequency. This is due to the compressibility of water contained air.

(2) When the performance of the single CD is compared with that of the double $\mathrm{CD}$, the efficiency of the single $\mathrm{CD}$ is better than that of the double CD.

(3) For the double CD, efficiency of chamber2 was better than that of chamber1, because the cross section area of chamber 2 is small and ratio of volume change is larger than that of chamber1.

(4) Characteristics of the CD applied in mass-damper-spring system was investigated and it was known that the colloidal damper had very complex nonlinear characteristics which is composed of damping force occurred by the colloid of silica gel and water, and Coulomb friction damping occurred by the V-packing, therefore the CD dissipated the energy in high frequency range but the piston did not move in low frequency range because of Coulomb friction occurred by V-packing. So it is necessary to reduce the friction force of the seal. From this reason very complex vibration could be observed. This will be analyzed in future.

\section{References}

(1) N.P.Chironis, Spring Design and Application, McGraw-Hill, pp204-242, 1961

(2) C.M.Harris and C.E.Crede, Shock and Vibration Hand book, McGraw-Hill, pp-1-418, 1982

(3) V.A.Eroshenko, Effect of Heat Exchange on Filling of Lyophobic Pores and Capillaries with Liquid, Colloid J., vol.49, No.5, pp875-880, 1995

(4) V.A.Eroshenko, Damper with High Dissipating Power, Patent WO 01/ 55616 A1, pp1-44, 2001

(5) A.Y.Fadeev and A.Eroshenko, Study of Penetration of Water into HYdrophobized Porous Silicas, J.Colloid and Interface Sc., vol.187, pp275-282,1997

(6) C.V.Suciu, T.Iwatsubo and S.Deki, Investigation of a Colloidal Damper, J.Colloid and Interface Sc., vol.259, No.1, pp62-80, 2003

(7) C.V.Suciu, T.Iwatsubo and S.Deki, Novel Principle of Mechanical Energy Dissipation (Part1: Static Performance of Colloidal Damper), JSME International Journal Series C, vol.47, No.1, pp180-188, 2004

(8) C.V.Suciu, T.Iwatsubo and S.Deki, Novel Principle of Mechanical Energy Dissipation (Part2: Dynamic Performance of Colloidal Damper), JSME International Journal Series C, vol.47, No.1, pp189-198, 2004

(9) T.Iwatsubo, C.V.Suciu, M.Ikenaga and K.Yaguchi, Dynamic Characteristics of New Damping Element Based on Surface Extension Principle in Nano Tube, Special Issue of the Journal of Sound and Vibration, Published in July, 2007

\section{Acknowledgements}

Authors would like to acknowledge supported by the Japanese Grant-in-Aid for Scientific Research No.16360117-5001. 